\title{
El interés superior del niño en el marco de la educación peruana
}

\author{
Mgtr. Amelia Dalia Toralva Mejía \\ atoralvam@ucvvirtual.edu.pe \\ ORCID: 0000-0001-5098-1116 \\ Universidad César Vallejo
}

\section{RESUMEN}

El objetivo de la investigación ha sido efectuar una revisión y análisis del principio Interés Superior del Niño, quienes, por su condición de vulnerabilidad, requieren de un tratamiento especial; para lo cual deberá articularse este principio jurídico con sector de Educación, garantizándoles así una educación de calidad, y al ejercicio pleno de sus derechos. Se recurrió a la base de datos Scopus, Scielo, Redalyc, Dialnet y Google Scholar, de forma gratuita y en español de los últimos 5 años. Método: En la metodología, se empleó para búsqueda y revisión de producción científica, términos como de: "Interés Superior del Niño", "La educación en el Perú", "El niño, sujeto de derechos", no considerando artículos trabajados en otro idioma que no sea el español, o que hayan sido publicados en el 2014 o antes o cuya descarga no sea gratuita. Resultados: Del análisis y discusión de cada uno de los artículos científicos, se estableció como una tarea prioritaria del Estado, velar por el Interés superior del niño, así como el de brinda una educación de calidad y la defensa de sus derechos.

Palabras Clave: interés superior del niño, educación; articulación. 


\title{
Child higher interest in the peruvian educational framework
}

\begin{abstract}
The objective of the research has been to carry out a review and analysis of the principle of the Best Interest of the Child, who, due to their vulnerability, require special treatment; for which this legal principle must be articulated with the Education sector, thus guaranteeing them a quality education, and the full exercise of their rights. The Scopus, Scielo, Redalyc, Dialnet and Google Scholar databases were used, free of charge and in Spanish for the last 5 years. Method: In the methodology, terms such as: "Best Interest of the Child", "Education in Peru", "The child, subject of rights", were used for the search and review of scientific production, not considering articles worked on in another language other than Spanish, or that have been published in 2014 or before or whose download is not free. Results: From the analysis and discussion of each of the scientific articles, it was established as a priority task of the State, to ensure the best interest of the child, as well as to provide a quality education and the defense of her rights.
\end{abstract}

Key Words: Higher interest of the child, education, articulation

Artículo recibido: 20 diciembre. 2021

Aceptado para publicación: 10 enero 2022

Correspondencia: atoralvam@ucvvirtual.edu.pe

Conflictos de Interés: Ninguna que declarar 


\section{INTRODUCCIÓN}

La evolución normativa de la protección del menor a través de la historia, lo encontramos en el derecho romano, donde estos no gozaban de ninguna protección legal, pues era el padre a través de la Tutela, quien protegía, cuidaba, velaba por su hijo, disponiendo de su vida y de su futuro; en la edad media, no se reconocía los derechos a los menores; a nivel internacional, a mediados del siglo XVIII y XIX, con el Derecho anglosajón se da por primera vez el concepto de bienestar del infante; asimismo en el derecho francés, el Estado asume algunos casos de la tutela del niño, protección al trabajo infantil, y al derecho a una educación gratuita y obligatoria, marcando el hito en beneficio de la humanidad. Es recién en el siglo XX, al amparo de las normas internacionales se le equipara como sujeto de derechos, dejando de ser objeto de posesión de sus padres (Rea 2016).

El principio del “interés superior del niño", consiste en la atención que el Estado brinda a la infancia para garantizar la protección de sus derechos, así como también al disfrute a una vida digna; a nivel internacional en 1924, con la Declaración de Ginebra, se instaura señalando que niño merece atención y cuidados, en 1934, tenemos el nuevo texto de la Declaración de Ginebra, referente a los derechos del menor; en 1959 se promulga la Declaración de los Derechos del Niño, sustentando el "Interés Superior del Niño" y que recién en 1989, la Convención de los Derechos del Niño, lo consagra como un principio, siendo recogida por los diferentes estados como derechos fundamentales, a fin de que logren alcanzar el bienestar posible, la armonía consigo mismo y con su entorno (Cabanillas, 2018).

Este aporte legal concedido a los menores de edad, surgió en América Latina, o también conocido como derecho angloamericano, donde el Interés Superior del niño, tiene carácter de norma fundamental y por lo tanto están jurídicamente protegidos sus derechos, y que por La Convención Internacional de los Derechos de niño (1989), dejaron definitivamente de ser considerados como propiedad de sus progenitores; para ser considerados como personas con derechos, integrante de una familia, con derechos y deberes, basados en los cuatro principios fundamentales a la no discriminación, el del interés superior del niño, el derecho a la vida, a la supervivencia y al desarrollo, y al respeto a la opinión del menor (Ravetllat, 2015). 
En el Perú, en 1990, se ratifica la Convención sobre los Derechos del Niño, otorgándoles protección y derechos desde su concepción, hasta los 18 años, donde adquiere la mayoría de edad, en 1992, se establece el Código del Niño y Adolescente, acogiendo el principio del Interés Superior del niño, así como las acciones o medidas que deben de cumplir para desterrar cualquier tipo de problemática o desafíos que vulneren los derechos de los menores (Ortiz, 2016).

Asimismo, en la Constitución de 1933, se establece la obligatoriedad de la enseñanza en la educación primaria; en la 1979, comprendió el reconocimiento de los instrumentos internacionales y ratificó los Derechos Humanos; protegiendo especialmente al niño y al adolescente; expuesto por Carpio y Pazo (2016). La Constitución de 1993, en su artículo $4^{\circ}$, por única vez y en forma breve, sucinta, lacónica, refiere a la protección del niño y a la familia, garantizando la efectividad del Principio del Interés Superior del Niño, como un derecho; por ello ninguna ley, propuesta legislativa o proyecto de ley, ni acto procesal o judicial, puede ignorar, desconocer, transgredir los derechos de los menores, normados y ratificados en la Convención (Perceval, 2019).

Vemos entonces que nuestra legislación está en constante cambio, donde se enfatiza tras la difusión de los derechos humanos, incluidos los menores de edad, de gozar de sus derechos establecidos y reconocidos y que por lo tanto es una obligación, un deber de los Estados de fomentar y asegurar su real protección igualitaria, en cumplimiento de este principio, que cautela su acatamiento en cada uno de sus derechos reconocidos (Cangas, 2019).

De igual forma el artículo 13 de nuestra Carta Magna de 1979, nos refiere sobre la trascendencia de la educación; por lo que urge en atender a la población estudiantil, que no está accediendo a este servicio adecuadamente, sugerido por Cuenca (2019). Y que dado a la emergencia sanitaria por la pandemia del COVID-19, la utilización de las Tics ha favorecido la continuación del proceso educativo, pero con las limitaciones en el uso de esta herramienta tecnológica (Acho, 2021) y como consecuencia de ello principalmente en las zonas más deprimidas de nuestra capital, así como de las zonas rurales se han visto acentuadas estas brechas digitales, expuesto por Anaya (2021); así como del escaso o nulo tiempo que tienen los padres de familia, en acompañar en sus estudios desde casa a sus hijos, (Bazán, 2021)

Por tales razones, el objetivo principal es articular el Principio del Interés Superior del 
Niño, como principio jurídico garantista, señalado por Bobadilla; con la obligación que tiene el Estado de brindar un servicio que garanticen una educación de calidad. La importancia de su articulación es para que el estudiante, logre alcanzar los aprendizajes para poder enfrentar los retos de la vida y ejercer una ciudadanía crítica, basado en valores. La justificación teórica, radica en el análisis de las investigaciones científicas provenientes de la fundamentación del principio del interés superior del niño y la realidad educativa actual en el Perú.

\section{METODOLOGÍA}

En este estudio, se siguió una metodología rigurosa de tipo revisión bibliográfica junto al análisis de contenido; posibilitó un estudio y revisión ordenada y minuciosa de los artículos científicos relacionados al tema de Interés Superior del Niño en el marco de la Educación Peruana, recurriendo a la literatura científica en revistas indexadas a nivel mundial, Latinoamericana y nacional y en los repositorios digitales de Scopus, Scielo, Redalyc, Dialnet y Google Scholar, hallazgos correspondientes al año 2015 al mes de diciembre 2021.

\section{El diseño y el sitio del estudio}

Se efectuó por medio de una revisión sistemática de artículos de producción científica, cuyo contenido incluía: nombre del autor o autores, año, título, fuente, DOI y referencias. La obtención de datos se inició en el noviembre 2021 y culminó en enero 2022.

\section{Criterios de elegibilidad}

- Tipos de participantes: El presente estudio se incluyó a los estudiantes de la Educación Básica Regular, que por su condición de vulnerabilidad; necesitan de una protección especial, basado en el principio del Interés Superior del Niño; habiéndose excluido aquellas revisiones de literatura de naturaleza teórica o que no estén inmersos en el tema desarrollado.

- Tipos de estudios: Teniendo en cuenta la escasa información de artículos científicos sobre El Interés Superior del Niño en el marco de la Educación Peruana, el objetivo se orientó en analizar los estudios desarrollados sobre el tema e identificando los artículos de revisión los diseños, de estudio, categorías, variables y dimensiones.

- Tipos de resultados:Se recopilo como resultados primarios los siguientes: casos de estudios, investigaciones empíricas, experiencias y similares, a fin de dar sustento al presente trabajo. 


\section{Búsqueda de estudios}

Se efectuó una exploración manual utilizando referencias de estudios primarios y secundarios hallados en la búsqueda electrónica. Se trabajó directamente con la base de datos Scopus, Scielo, Redalyc, Dialnet y Google Scholar, para lo cual se tuvo como criterio la consulta por títulos, resúmenes y por las palabras claves "El interés superior del Niño", "La educación en el Perú”, "El niño, sujeto de derechos" y "La educación en tiempos de pandemia”.

\section{Selección de estudios}

El investigador realizó el proceso de selección de los diferentes artículos científicos encontrados. El trabajo de selección de los artículos científicos, se efectuó en dos etapas. El primer paso se procedió a revisar los títulos y resúmenes de las referencias encontradas, aplicando diversas estrategias de búsqueda, seleccionándose los estudios potencialmente elegibles. El segundo paso, para confirmar su elegibilidad, se procedió a analizar, revisar el texto completo de los artículos preseleccionados.

\section{RESULTADOS}

Las estrategias de búsqueda arrojaron 45 artículos. Durante el proceso de selección no se encontraron referencias duplicadas, eliminándose 30 artículos que no cumplieron los criterios de inclusión tras revisar el título, el resumen o palabras clave (primer paso). Los 15 artículos seleccionados fueron leídos para confirmar la elegibilidad (segundo paso). Asimismo, se obtuvo información sobre las bases consultadas, analizándolos 15 artículos seleccionados de Scielo, con 06 artículos lo que equivale al 40\% y de Dialnet 1, Ecociencia 1, Episteme 1, Lantindex 1, Redalyc 1, Reice 1, Revista Instituto de la Familia UNIFE 1, Revista de Investigación de la UNMSM 1, que equivale a un 7\% respectivamente a cada uno de revistas enunciadas. (ver figura 1) 
Figura 1. Artículos según base de datos.

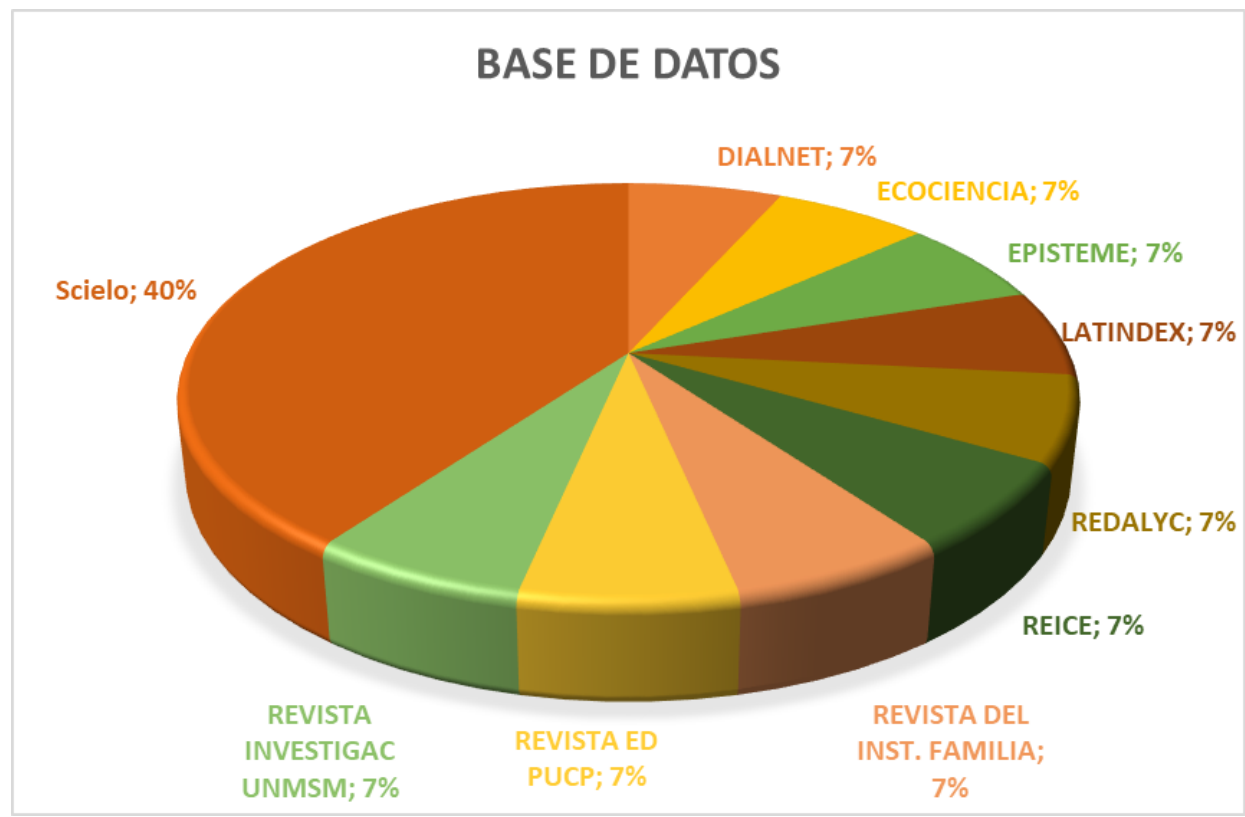

Además, se ha graficado los 15 artículos por año de publicación, habiéndose ha obtenido el siguiente resultado: Del 2021, se tiene 4 artículos que equivale al 27\%; del año 2020, se tiene 3 artículos que equivale al 20\%; del 2019, se tiene 2 artículos que equivale al 13\%, del 2018, se tiene 2 artículos que equivale al 13\%, del 2016, se tiene 3 artículos que equivalen $20 \%$ y del 2015, se tiene 1 artículo que equivale al $7 \%$ (ver figura 2)

Figura 2: Artículos según años de publicación

\section{ARTÍCULOS SEGÚN AÑOS DE PUBLICACIÓN}

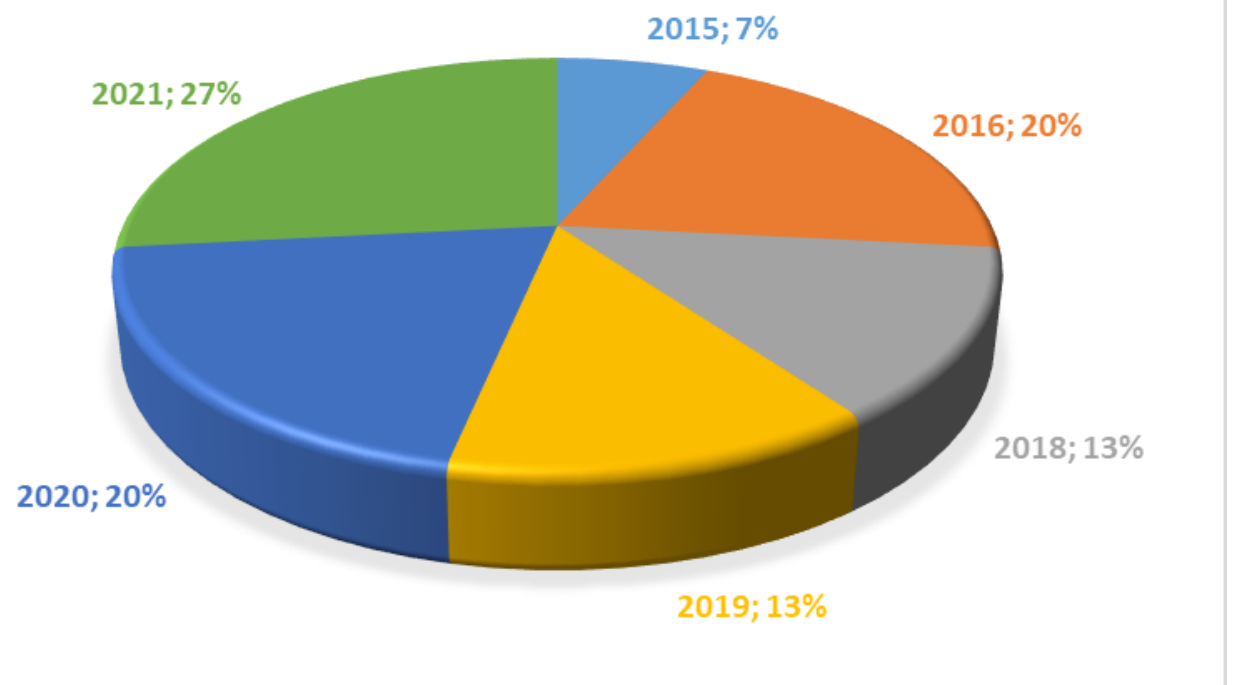

De la misma forma, se procedió a las ubicaciones por países de los 15 artículos, siendo 
que Perú ha publicado 9 artículos que equivale a un 60\%, España tiene 2 artículos que equivale al 13\%, Ecuador tiene 2 artículos que equivale al 13\%, México 1 y Chile 1 que equivale al $7 \%$ respectivamente cada uno. (ver figura 3 )

Figura 3. Países que publicaron los artículos

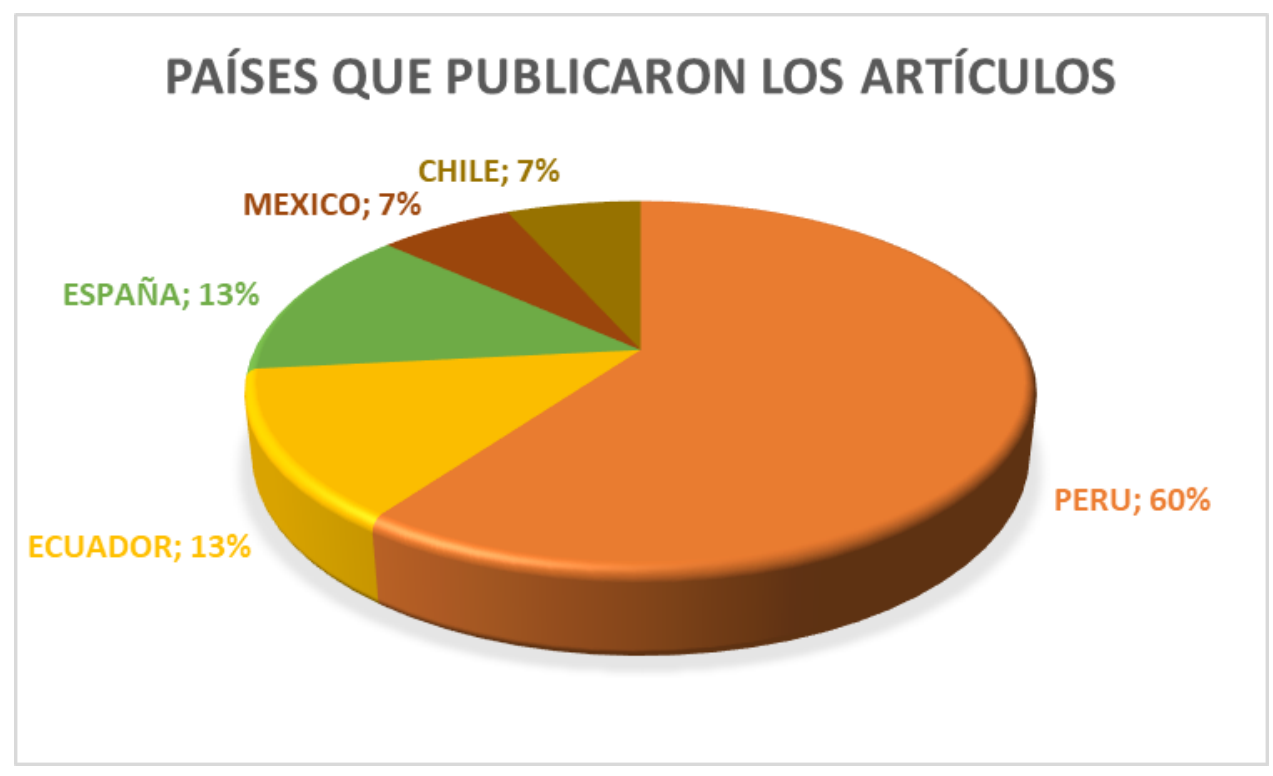

Igualmente, de la revisión de los 15 artículos de los textos completos seleccionados, se presentan a continuación de manera descriptiva, ya que la naturaleza de estos estudios no permite ningún otro tipo de análisis de igual manera, la tabla 1 presenta los resultados de manera concisa.

A continuación, presentamos a los autores de textos y revistas indexadas, cuyos contenidos fortalecen el tema principal:

El primer artículo, presentado por Acho et al. (2021), es una investigación original, desarrollada en el Perú, el método empleado fue del análisis de contenido. Entre sus resultados sostuvo que la virtualidad brinda la posibilidad de flexibilizar la educación presencial, integrando de forma activa a los padres de familia o familiares para que den la orientación, el apoyo que necesita el menor, para llevar a cabo sus actividades propias de su etapa escolar. Concluyó, que un problema critico en el Perú, es la poca información de los grupos de estudiantes en condiciones de desigualdad o exclusión del alumno, a fin de detectar su vulnerabilidad o carencias que no le permitan seguir con éxito sus estudios. La importancia del estudio, radica en señalar que los entornos virtuales proporcionan la posibilidad de adaptar en estos tiempos de pandemia, y llegar a los grupos menos 
favorecidos.

El segundo artículo, realizado por Anaya et al. (2021), corresponde a una investigación original, desarrollada en el Perú, el método trabajado fue de tipo cualitativo, analítico descriptivo. Entre sus resultados, consideraron que las brechas digitales están asociadas a la escasa o nula conectividad, así como también a dispositivos móviles pre pago y de poca capacidad, y que sus viviendas son precarias y carentes de los servicios elementales como de agua y luz; por lo que se debe de enfrentar las desigualdades desde su origen. Concluyó que ante tales falencias el gobierno central, debe prioritariamente atender al sector de educación a fin de cortar las brechas digitales existentes. La importancia del estudio fue en evidenciar la necesidad de potenciar la conectividad para enfrentar las desigualdades sociales y educativas que se dan en nuestro medio.

El tercer artículo, analizado por Bazán, et al. (2020), es una investigación original, desarrollada en Costa Rica, el método trabajado fue de tipo cualitativo fenomenológico. Entre sus resultados, manifestaron que no se debe de establecer tiempo y que el apoyo o la supervisión del aprendizaje del menor debe de ser permanente. Concluyeron, que el apoyo familiar es el pilar en el desarrollo educativo del menor a fin de lograr que crezca y desarrolle su formación integral y con ello formar al futuro ciudadano crítico y con valores. La importancia del estudio fue fortalecer la integración familiar a fin de prodigar al menor una verdadera formación.

En el cuarto artículo planteado por Tarazona, N., (2021), corresponde a una investigación original realizada en la Universidad Católica de Chile; el método trabajado fue el análisis de contenido documental. Entre sus resultados, considera que la pandemia traslució muchas deficiencias a nivel mundial respecto al proceso de la enseñanza virtual. Entre sus principales conclusiones, menciona que ante la emergencia sanitaria, nos obliga a mirar, adecuarnos e innovar para lograr la transformación digital en la educación. La importancia del estudio fue dar énfasis a la enseñanza virtual, la cual deberá ser más motivadora y flexible, para lograr captar el interés de todos los estudiantes.

El quinto artículo, realizado por Bobadilla (2020), es una investigación original, desarrollada en el Perú, el método trabajado fue de tipo cualitativo analítico descriptivo. Entre sus resultados, afirma que el Interés superior del niño es un principio jurídico garantista, entendiéndose como el deber, que tiene toda autoridad de cautelar los derechos de los menores. Se concluyó, que todas las acciones del Estado o de cualquier entidad 
pública o privada deben de estar orientadas a la protección del menor, cautelando todos sus derechos que por ley le corresponden. La importancia, del estudio, fue resaltar el rol del Estado, como el primer ente en cautelar los derechos del menor.

El sexto artículo planteado Cotino (2020), corresponde a una investigación original, desarrollada en Colombia, el método trabajado fue de tipo cualitativo, analítico descriptivo. Entre sus resultados, enfatiza que ante la presencia de la pandemia la educación virtual ha sido un salvavidas que garantizó la continuidad de la enseñanza. Se concluyó que frente a esta crisis sanitaria que para algunos fue comparada como un tsunami que rompió paradigmas y facilito la adecuación y aceptación de las TIC, en la continuidad de la educación. La importancia del estudio fue establecer la trascendencia que debe de tener la educación digital, y que debe de ser garantizada constitucionalmente. El séptimo artículo propuesto Murillo et al. (2020), es una investigación original, desarrollada en Madrid, España, el método trabajado fue de tipo cuantitativo. Entre sus resultados, se desprende al señalar que todo investigador debe de ser neutral, transparente y sobre todo leal, verídico con sus resultados, a fin de acabar con las injusticias e inequidades principalmente para aquellos menos favorecidos. Se concluyó que no se debe de vulnerar ningún derecho y menos de los menores, a recibir una educación de calidad. La importancia del estudio fue en recomendar que toda investigación de índole social debe ser lo más fidedigno a la realidad de la población en estudio y solo así se podrá proponer o hacer cambios a fin de acortar dichas brechas.

El octavo artículo, propuesto por Cangas et al. (2019), es una investigación original, desarrollada en Ecuador, el método trabajado fue de tipo inductivo, deductivo, analítico y sintético para revelar los aportes técnicos. Entre sus resultados, argumentan que el Estado, la sociedad y la familia, son quienes deben de promover de manera asertiva el desarrollo integral de los menores. Se concluyó enfatizando que este principio representa la esencia misma de la protección al menor. La importancia del estudio fue demostrar la supremacía legal de este principio a fin de proteger al menor, haciéndolo obligatorio su cumplimiento.

El noveno artículo, señalado Cuenca et al. (2019), es una investigación original, desarrollada en el Perú. El método trabajado fue un análisis cualitativo. Entre sus resultados mostraron que las desigualdades educativas en el país continúan, pese a los esfuerzos que el gobierno realiza para contener este avance. Concluyó argumentando que 
debido a la trascendencia que tiene la educación para todo pueblo, es urgente atender a las poblaciones que no están accediendo adecuadamente a este servicio, y que están generando una brecha de desigualdad en el logro educativo. La importancia del estudio fue mostrar las desigualdades educativas que imposibilitan el éxito en el área educativa. El décimo artículo presentado por Cabañilla (2018), es una investigación original, desarrollada en el Perú, el método trabajado fue de tipo análisis de contenido documental. Entre sus resultados, plantea que se debe de defender el Principio del Interés Superior del niño, en cualquier circunstancia, donde el menor se vea afectado, incluyendo en el tema de la adopción. Se concluyó que tanto el Estado, la sociedad y la familia deben de asegurar, el cumplimiento de la normatividad de protección del menor y avalar el disfrute pleno, real de todos sus derechos. La importancia del estudio fue, en sostener que se debe de proteger al menor a través del cumplimiento de las normas establecidas a su favor. El onceavo artículo declarado por Gómez (2018), es una investigación original, desarrollada en Chile, el método trabajado fue de tipo cualitativo, analítico descriptivo. Entre sus resultados, establece que, de acuerdo a la Convención de los Derechos del Niño, deben de ser considerados como sujetos de protección por parte de sus padres y del Estado. Se concluyó que el menor debe ser sujeto de derechos y deberes de acuerdo a su edad cronológica y su avance y madurez. La importancia, del estudio fue demostrar que el menor sea sujeto de derecho y obligaciones, así como el de ser escuchado a fin de conocer cuáles son sus sentimientos e intereses.

El doceavo artículo presentado Ortiz et al. (2016), es una investigación original, desarrollada en el Perú, el método trabajado fue de tipo cualitativo, analítico descriptivo. Entre sus resultados, que el Perú incorpora entre su legislación los Derechos del Niño y del adolescente, a fin de protegerlos ante cualquier reto o desafío que se le pudiera presentar. Se concluyó que el Estado peruano, hace suya dentro de su normatividad la Declaración de los Derechos Humanos y por ende los derechos a favor del menor. La importancia, del estudio, fue el de salvaguardar los derechos de los menores ante cualquier realidad que le sea adversa.

El treceavo artículo, declarado por Carpio et al. (2016), es una investigación original, desarrollada en el Perú, el método trabajado fue de tipo inductivo, deductivo, analítico y sintético para revelar los aportes técnicos. Entre sus resultados, Sostiene que, la historia de la república, se dio inicio con la Proclamación de la Independencia del Perú en 1821, 
concatenándose hechos o sucesos políticos o sociales que marcan la evolución del derecho constitucional. Se concluyó, que el Perú, a través de la Constitución de 1979, contemplo a la persona, como el fin supremo de la sociedad y el Estado, reafirmando su contenido de la Convención Internacional de los Derechos del Niño. La importancia del estudio fue consagrar el reconocimiento de diversos derechos constitucionales que regulan los derechos a favor del menor.

El catorceavo artículo mencionado por Rea (2016), corresponde a una investigación original desarrollada en Colombia. El método trabajado fue un análisis cualitativo. Entre sus resultados destaco que su objetivo fue detallar la evolución histórica de los derechos de los menores y su reconocimiento internacional, garantizados mediante instrumentos internacionales. En sus conclusiones, sostuvo que, el proceso del reconocimiento de los derechos de los menores no termino con la adopción de la Convención sobre Derechos del Niño, sino que continua con la implementación de este principio. La importancia de este estudio, fue en demostrar la evolución internacional de los derechos del menor y la implementación de normas, principios y derechos a favor de este.

En el quinceavo artículo referido por Ravetllat et al. (2015), es una investigación original expuesta en la Universidad Católica de Chile; el método trabajado fue el análisis de contenido documental. Entre sus resultados, consideraron que la conceptualización del interés superior del niño, se fundamenta en un sistema normativo, donde se deberá respetar cada una de las resoluciones; brindándosele una solución justa, real y efectiva a favor del interés superior del menor. Entre sus principales conclusiones, afirmaron que el niño y adolescente debe de ser reconocidos como personas y como ciudadanos y como tal deben de gozar de todos sus derechos fundamentales acordes a su edad. La importancia del estudio fue detallar como este principio ha evolucionado y consolidado en el marco internacional, como un principio rector a favor de la niñez.

\section{DISCUSION Y CONCLUSIONES}

El interés superior del niño, es un principio rector que tiene por objetivo proteger, cautelar, el desarrollo del menor; así como también busca salvaguardar situaciones de hecho donde pudieran estar inmersos niños, niñas y adolescentes, a fin de prodigarles su bienestar en la satisfacción de sus necesidades; motivo por el cual se debe de considerar que en tales circunstancias al menor como sujeto de derechos por lo que debe de ser oído y considerar su opinión cuando este frente a una situación que le afecte, la cual deberá ser 
contemplada de acuerdo a su edad y grado de madurez, dejando de ser un sujeto pasivo de protección, coincidiendo con lo sustentado por Gómez (2018). Y que a su vez se encuentra respaldado por Torrecuadrada (2016), al sostener que el Interés superior del menor es un principio de estímulo, de inspiración para la real protección del menor debido a su situación de vulnerabilidad.

El objetivo, es articular el Principio superior del niño con la educación peruana, conceptualizándolo como el proceso por el cual se desarrolla habilidades, actitudes durante su aprendizaje (Tesauro de la Unesco) y considerándosele como un derecho para todas las personas y cuyo sustento lo encontramos en la Declaración Universal de Derechos Humanos, la Convención de la ONU; contrastando con la realidad donde los estudiantes pobres o de extrema pobreza, evidencian una segregación escolar por su nivel socio económico, por lo que su derecho a la educación ha sido vulnerado; tal como lo ha señalado Murillo (2019); es decir desaparece o se ve lejano para sus aspiraciones y para las metas de nuestro país de querer posesionarnos como un país emergente; pese a que nos hemos visto afectado por la emergencia sanitaria, del COVID-19, que ha agudizado nuestra problemática educativa, que nos obliga a todos a adecuarnos e innovar para lograr la transformación digital en la educación, también lo plantean Cotino L. (2020) y Tarazona (2021).

De las investigaciones revisadas coinciden que el Estado debe de generar políticas públicas para enfrentar este reto, y articular al sector educación y con el de justicia, dándosele un carácter transversal a este principio dentro de la política educativa; a fin de que favorezca e impuse la formación integral de los estudiantes, y con ello el desarrollo del país; dado que los beneficiarios de esta investigación son los niños, niñas y adolescentes, inclusive se sugiere la apertura de una nueva especialidad denominada Derecho Educativo, tan igual como existe con otras especialidades y solo así profesores, operadores de justicia, harán cumplir este principio en favor del menor, del estudiante en el Perú y con ello dar el despegue a la educación y por ende al país.

\section{CONCLUSIONES}

De los resultados de la revisión sistemática del presente trabajo titulado Interés Superior del Niño en el marco de la Educación Peruana, se concluye que:

PRIMERO: El avance de los derechos de la infancia doctrinalmente, se ubica en primera instancia desde la desprotección, la indiferencia, el olvido hasta llegar a consolidarse 
como un derecho real, motivado por razones de carácter humanitario y social, hasta llegar a consolidarse, con el principio fundamental del Interés superior del niño, protegiéndosele jurídicamente ante cualquier situación en contrario que pueda vulnerar sus derechos.

SEGUNDO: Analizar e investigar la realidad del Perú, donde se deberá identificar a los grupos que son objeto de la desigualdad social y de la exclusión, principalmente en el ámbito educativo, a fin de poder identificar esta problemática, llegando a estos sectores brindándosele la atención preferente, promoviendo la educación como derecho fundamental a lo largo de la vida.

TERCERO: El interés superior del niño, se cristaliza, con el ejercicio progresivo de sus derechos, es decir cuando el menor es sujeto de derechos, inclusive a ser oído y dar su opinión con buen criterio de la apreciación de sus facultades.

CUARTO: El inicio, el origen, de las Naciones Unidas, fortaleció y consolido los derechos humanos, como eje rector que debe de orientar, dirigir, encaminar a la toda la sociedad a nivel nacional e internacional, en beneficio del menor, que y que el Perú lo ha ratificado en la Convención de los Derechos del Niño en 1989.

QUINTO: Todo investigador debe de efectuar una visión panorámica de la realidad en estudio, la cual deberá ser verídica, neutral a fin de incentivar, contribuir a revertir esta realidad en beneficio de los menos favorecidos de un sistema educativo clasista que afecta y agobia al menor en edad escolar; proponiendo acciones que conlleven al respeto de los derechos del menor y por ende a tener una sociedad justa y equitativa.

SEXTO: Ante la presencia de la emergencia sanitaria, a causa del COVID-19 y a fin de dar continuidad al sistema educativo, tuvo una rápida reacción, aplicar la estrategia digital, para llegar a todos los estudiantes mediante el programa de Aprendo en casa, cuya difusión fue por radio, televisión y plataforma web, pese a que tuvo muchas falencias, debe de ser preocupación del Estado, masificar estas herramientas tecnológicas, para el desarrollo de una educación de calidad.

SEPTIMO: Que el Estado, así como cualquier entidad pública o privada, debe de tener como pilar en todas sus actuaciones al Principio del Interés Superior del Niño, la cual tiene carácter garantista, y por lo tanto como responsabilidad el de cautelar los intereses del menor, salvaguardando su bienestar y derechos frente a cualquier otra pretensión en contrario, articulándose con el sector de educación, como un eje transversal, para otorgársele una educación de calidad, sin ningún tipo de discriminación. 


\section{REFERENCIAS}

Acho, S., Díaz, M., Criollo, V., \& García, O. (2021). La realidad de la educación inclusiva en el Perú y los retos desde la virtualidad. EduSol, 21(77), 153-168. Epub 18 de octubre de 2021. Recuperado de http://scielo.sld.cu/scielo.php?script=sci_arttext\&pid=S1729-

$80912021000400153 \& \operatorname{lng}=$ es \&tlng=es.

Anaya, T., Montalvo, J. Calderón, I \& Arizpe, C. (2021). Escuelas rurales en el Perú: factores que acentúan las brechas digitales en tiempos de pandemia (COVID-19) y recomendaciones para reducirlas*. Educación, 30(58), 11-33. Recuperado de https://dx.doi.org/10.18800/educacion.202101.001

Bazán, A., Márquez, L., Guadalupe, E., (2021). Apoyo familiar en el estudio de escolares en un contexto de vulnerabilidad. Revista de Educación, Vol. 46, No. 1, 2022, Universidad Federico Villarreal. Recuperado de https://revistas.ucr.ac.cr/index.php/educacion/article/view/44903/49100

Bobadilla, M., (2020) Normativa laboral para adolescentes en el Perú y la eficacia normativa. Desde el Sur vol. 12 No. 1, Lima, ene/jun2020. Recuperado de http://www.scielo.org.pe/scielo.php?pid=S2415-

09592020000100127\&script=sci_arttext

Cabanilla, J. y Caveda D. (2018), Las adopciones tradicionales y la vulneración del principio del Interés Superior del Niño. Revista Científica ECOCIENCIA. Ecuador Recuperado de https://revistas.ecotec.edu.ec/index.php/ecociencia/article/view/2/1

Cangas, L; Iglesias, J y Mosquera, M, y Puerta, Y. (2019). El interés superior del niño y el estricto respeto al principio de la convencionalidad de las normas. Artículo científico. Ciencias Sociales. Revista digital de Ciencia, Tecnología e Innovación. $\begin{array}{llll}\text { Inicio } & \text { Vol } & 6 & \text { Ecuador. }\end{array}$ http://45.238.216.13/ojs/index.php/EPISTEME/article/view/1774/1027

Carpio, E. y Pazo, O. (2016). Revista de Derecho VOX JURIS Temas de Derecho Constitucional. Edición 31, Universidad San Martin de Porres. Facultad de Derecho. Latindex. Evolución del Constitucionalismo Peruano. Pág. 29. Recuperado de https://derecho.usmp.edu.pe/biblioteca/vox_jurix/vox_juris_31.pdf 
Código de los Niños y Adolescentes. Ley $\mathrm{N}^{\mathrm{a}}$ 273337. Recuperado de https://www.mimp.gob.pe/files/direcciones/dga/nuevo-codigo-ninosadolescentes.pdf.

Cotino, L., (2020), La enseñanza digital en serie y el derecho a la educación en tiempo del coronavirus. Universidad de Valencia. Revista de educación y derecho, ISSN 2013-584X, No. 21. Recuperado de https://dialnet.unirioja.es/servlet/articulo?codigo=7388655.

Constitución Política del Perú - 1993. Recuperado de http://www.pcm.gob.pe/wpcontent/uploads/2013/09/Constitucion-Pol\%C3\%ADtica-del-Peru-1993.pdf

Convención sobre los Derechos del Niño. Plataforma de infancia. España. Recuperado desde https://plataformadeinfancia.org/derechos-de-infancia/convencionderechos-del-

nino/?gclid=Cj0KCQiAxc6PBhCEARIsAH8Hff3ZrqgHk6Vf0ea7J_KaxR5c9S9 g-381r4UCi2fXQeqK12zhYXN7YLEaAhH9EALw_wcB

Cuenca, R, y Urrutia, C. (2019). Explorando las brechas de desigualdad educativa en el Perú. Revista mexicana de investigación educativa, 24(81), 431-461. Recuperado en 09 de diciembre de 2021. Recuperado de http://www.scielo.org.mx/scielo.php?script=sci_arttext\&pid=S1405$66662019000200431 \& \operatorname{lng}=$ es\&tlng=es.

Gómez, M. (2018). Las implicancias de considerar al niño sujeto de derechos. Revista de Derecho (Universidad Católica Damasco A. Larrañaga, - Chile. Facultad de Derecho) versión impresa ISSN 1510-3714 versión On-line ISSNN 2393-6193. Rev. Derecho No. 18 Montevideo dic. 2018. Recuperado de http://www.scielo.edu.uy/scielo.php?script=sci_arttext\&pid=S2393$61932018000200117 \&$ lang=es

Guadalupe, C; Rodríguez, J; Vargas, S. (2017), Estado de la educación en el Perú: análisis y perspectivas de la educación básica.: FORGE Fortalecimiento de la gestión de la educación en el Perú. Recuperado de https://www.grade.org.pe/publicaciones/estado-de-la-educacion-en-el-peruanalisis-y-perspectivas-de-la-educacion-basica/

Murillo, J., Graña, R., (2020), ¿Segregación escolar por nivel socioeconómico o por nivel de estudios de los Padres? Universidad Autónoma de Madrid, España. REICE. 
Revista Iberoamericana sobre Calidad, Eficacia y Cambio en Educación. 2020, 18

Recuperado

de

https://revistas.uam.es/reice/article/view/reice2020_18_4_001/12549

Ortiz Rosas, Carlos, (2016), Reflexiones sobre la Convención Internacional de los

Derechos del Niño, Vol. 1 Núm. 52016 Persona y Familia; Revista del Instituto de Familia. UNIFE.PE. Recuperado de https://revistas.unife.edu.pe/index.php/personayfamilia/article/view/461/247

Perceval M. C. Directora Regional UNICEF para América Latina y el Caribe. Adecuación normativa a la Convención sobre los Derechos del Niño en América Latina. Avances y deudas con la niñez. Panamá, agosto de 2019. Recuperado de https://www.unicef.org/lac/media/9646/file/PDF\%20La\%20adecuacion\%20nor mativa $\% 20 \mathrm{a} \% 201 \mathrm{a} \% 20$ Convencion $\% 20$ sobre $\% 201$ os $\% 20$ Derechos $\% 20 \mathrm{del} \% 20 \mathrm{~N}$ ino\%20en\%20America\%20Latina.pdf

Ravetllat, I, y Pinochet, R. (2015). El interés superior del niño en el marco de la convención internacional sobre los derechos del niño y su configuración en el Derecho Civil chileno. Revista chilena de derecho, 42(3), 903-934. Recuperado de https://dx.doi.org/10.4067/S0718-34372015000300007 https://www.scielo.cl/scielo.php?script=sci_arttext\&pid=S0718$34372015000300007 \&$ lang=es

Rea-Granados, S (2016) Evolución del Derecho Internacional sobre la Infancia, 29 International Law, Revista Colombiana de Derecho Internacional, 147-192 julio diciembre (2016). Recuperado de

http://www.scielo.org.co > pdf > ilrdi > 1692-815...

Tarazona, N., (2021), Tensión respecto a la brecha digital en la educación peruana. Revista peruana de investigación e innovación educativa, agosto 2021, Vol. 1, Núm. 2, e21039 Universidad Nacional Mayor de San Marcos, Facultad de Educación. On line

ISSN:

2789-0880.

Recuperado

de https://revistasinvestigacion.unmsm.edu.pe/index.php/repiie/article/view/21039/ 17088 